\title{
In Harm's Way
}

\author{
David Muller, MD
}

Medical Education, Mount Sinai School of Medicine, New York, NY, USA.

$\mathrm{W}$ ith my finger lodged firmly between the patient's tightly clenched teeth, I did what any other self-respecting resident would do; I panicked.

It was 3:00 a.m. and I was alone in a respiratory isolation room at the far end of a seemingly endless corridor with the patient we had just admitted. He had presented with "end-stage AIDS, fever and altered mental status," though "no mental status" described him more accurately. 'Ring-enhancing' lesions on his brain CT accounted for his clinical appearance upon his arrival from the ER; he was barely responsive. It was 1993 and at that point in my second year of residency, this was depressingly familiar. He had CNS Toxoplasmosis until proven otherwise, with lymphoma and tuberculosis also on the differential.

In that moment, however, these clinical details were far from my mind as I realized just how alone I was. The nurses on the ward had been asleep for hours (yes, asleep-this was a city hospital in the early 1990s), and my intern was elsewhere, drowning in admission notes and orders, putting out fires large and small.

How did I let this happen? I had allowed myself to end up in this remote room, in this frenetic hospital, with this very HIVinfected patient, whose teeth were now digging their way through my glove, and possibly my skin. His cachexia, Kaposi's sarcoma and known intravenous drug use were evidence enough that he was teeming with HIV and very likely hepatitis, as well.

During medical training, we're expected to acquire skills and perform tasks that sometimes require a healthy dose of irrational behavior. One must suspend disbelief when asked to examine a patient with active tuberculosis, or a patient who is agitated and combative. How about working in a bloody surgical field on behalf of a patient with advanced HIV/AIDS? Or simply staying up all night, fighting off exhaustion, while covering scores of sick patients and admitting new ones from the emergency room? We compromise relationships with family and friends, eat nothing but pizza for days on end, gain or lose more weight than we thought possible. For the sake of meaningful contact with patients and our ability to perform lifesaving procedures, we know that certain things must be sacrificed. That is, after all, the attribute we call altruism, and what some say makes us a more noble profession than most. But how far are we expected to go?

Model resident that I was, I had told my intern that I would take care of inserting our patient's nasogastric (NG) tube while she finished her 'scut' for the night. I wanted to teach her two golden rules: (1) a resident always pulls his weight when things get busy, and (2) the on-call team NEVER signs out an NG tube to the team coming in; it was simply bad form. Besides, an NG

Published online October 25, 2011 tube would take me no more than 5 minutes, and she was likely to struggle with it for half an hour. It seemed like an eminently good triage decision on my part; we'd both get to bed earlier this way.

I gathered up the tube, some gloves, lubricant, surgical tape, a bulb syringe to generate that satisfying gurgling sound confirming that the tube was actually in place, and a large disposable pad so as not to incur the wrath of the slumbering nurses if I made a mess.

An NG tube is arguably one of the most uncomfortable procedures a conscious patient can endure. Snaking two feet of tubing into someone's nose, down their throat, and into their stomach, especially when they're already sick and afraid, can be horrifying. It's painful-patients gag, sometimes vomit, and there can be lots of bleeding. Once in a while the tube enters the mouth instead of heading further south into the esophagus, coiling around behind the teeth while the patient quietly panics. Then you've got to pull the whole thing out and try again.

And yet we train at a stage of life when we're feeling invincible; we are pressed for time; we like to please, particularly our superiors and those evaluating us. We like a challenge for its own sake, sometimes forgetting that there's a patient on the other end of that needle, or scalpel, or tube. We are immersed in a culture of 'see one, do one, teach one.' All of these things encourage us to take unnecessary risks, to get in harm's way.

This, however, was going to be a piece of cake. My patient was so deeply unconscious he wasn't likely to feel anything, and as a second year resident, I felt my technique was impeccable.

I entered the room, set up my equipment on his lap, and positioned him by raising the head of his bed to 90 degrees. I kept the lights low. I still remember the window rattling in its futile attempt to keep the frigid December wind out of the room.

His head was covered with sparse, downy hair, his dry flaky skin stretched tight over his bony skull. His mouth was gaping open as if he was already dead. I flexed his neck by pressing my right hand against the back of his head so that his chin almost touched his chest, his mouth still slack. With that hand, I could feel the intense, dry heat of his fever even through my glove.

I dipped the tip of the tube in lubricant and inserted it into his nose. At first, things went smoothly. But when the tube

\footnotetext{
J Gen Intern Med 27(8):1090-1

DOI: $10.1007 / \mathrm{s} 11606-011-1907-2$

(C) Society of General Internal Medicine 2011
} 
approached his epiglottis, he reacted reflexively by biting downhard. The fourth finger on my left hand had been jutting out in much the same way that one's pinky might when taking high tea, perilously close to his mouth every time I advanced the tube. Now it was in between his teeth and he seemed to be clamping down harder by the second.

Excruciating, throbbing pain, an NG tube still dangling from my patient's nose, no way to call for help and no way to pry open his mouth with my one free hand. Panic might be an understatement. For the next few seconds my mind raced with a flurry of desperate thoughts: Was he going to bite my finger off? Was I going to end up with HIV? How do I get it out?!

I could try to describe what I did next in the context of some vaguely remembered medical school lesson about a primitive reflex that leads to sudden mouth-opening, but I would be lying. What I actually did was the unthinkable. I brought my right fist down on my patient's forehead as hard as I could. The shock of that blow caused him to suddenly open his mouth, allowing me to remove my finger and leap away from his bedpanting, my heart racing, my back covered in a cold sweat. He seemed no worse for the wear; mouth still slack, NG tube partially inserted. He slipped back into his stupor and resumed his quiet, rhythmic breathing.

We harm patients all the time in medicine, always regrettably, and presumably for the sake of beneficence in the long run. While patients rightly remain the focus of our attention, we are beginning to appreciate the extent to which we also harm ourselves, and the fact that sometimes we walk a fine line between our altruism and the need for self-preservation.
Sleep deprivation and inadequate nutrition are rites of passage for physicians. We have just begun to address the silent epidemic of depression, anxiety, eating disorders and other mental illness in our ranks. But do we also implicitly accept a degree of physical harm when we set our sights on a career in medicine?

I can personally boast of one bitten finger, one high-risk needle stick, two bouts of scabies, one MRSA cellulitis, and a well-placed, but thankfully weak left hook to my jaw from a dying, sofa-bound, former Merchant Marine during a home visit. I reported none of these incidents and my experience is not exceptional; over the years I've counseled many students and residents after errant needles, outbursts in the OR, abusive faculty, or angry families left them feeling unhinged and insecure.

Some harm is unavoidable and may even be constructive, providing us the opportunity to learn important skills in critical settings. How many people do you know who can wake up from a dead sleep and manage a medical crisis over the phone? But much harm can be prevented by being more aware of our surroundings and more comfortable functioning in teams. In our efforts to master the science and art, technology and humanity of medicine, it's easy to lose sight of another golden rule; there's no place here for bravado and rugged individualism. It's all about knowing your own limits and having the self-confidence to call for help when you need it.

Corresponding Author: David Muller, MD; Medical Education, Mount Sinai School of Medicine, One Gustave L. Levy Place, Box 1255, New York, NY 10029, USA (e-mail: David.muller@mssm.edu). 